\title{
PERFIL SOCIODEMOGRÁFICO E DE SAÚDE DE DIABÉTICOS ATENDIDOS EM CONSULTA DE ENFERMAGEM EM AMBULATÓRIO DE HOSPITAL UNIVERSITÁRIO
}

\section{SOCIODEMOGRAPHIC AND HEALTH PROFILE OF DIABETICS ASSISTED IN NURSING CONSULTATION IN AMBULATORY OF UNIVERSITY HOSPITAL}

\author{
Eliana Sayuri Seki ${ }^{1}$ (D), Ana Karina Silva da Rocha Tanaka ${ }^{*}$ (D), Maria Luiza Paz Machado ${ }^{1}$ (D), \\ Renatta Rossatto de Araújo ${ }^{1}$ (iD, Guilherme Breitsameter ${ }^{2}$ (iD) \\ ${ }^{1}$ Universidade Federal do Rio Grande do Sul, Porto Alegre, RS, Brasil. \\ ${ }^{2}$ Pontifícia Universidade Católica do Rio Grande do Sul, Porto Alegre, RS, Brasil. \\ *anakarinatanaka@gmail.com
}

\section{RESUMO}

As doenças crônicas não transmissíveis (DCNT), em especial o Diabetes Mellitus (DM), são um grave problema de saúde pública, sendo a consulta de enfermagem (CE) um instrumento utilizado no controle da doença e seus agravos. Estudo baseado em experiências de $\mathrm{CE}$ a pacientes portadores de DM em ambulatório de estabelecimento de saúde de nível terciário, hospital universitário geral de capital do sul do Brasil. O conhecimento da situação social, econômica e de saúde dos pacientes atendidos é uma forma de auxiliar o enfermeiro na obtenção de melhor resposta as combinações e orientações melhorando o controle da doença e incrementando a qualidade de vida do paciente. Teve como objetivo caracterizar o perfil sociodemográfico e de saúde de pacientes diabéticos atendidos em consulta de enfermagem em ambulatório de hospital universitário. Estudo de caráter quantitativo, delineamento transversal. A amostra do estudo foi composta por pacientes adultos, diabéticos, com primeira CE na agenda Enfermagem Adulto Diabético entre 2016 e 2017. A análise dos dados mostrou o predomínio do sexo feminino, brancos, casados, naturais do interior do estado, residentes na capital e região, baixa escolaridade e com grande número de faltas às consultas. Na saúde, observou-se: comorbidades, níveis glicêmicos e de hemoglobina glicada fora das metas terapêuticas. Conclui-se que o desafio em auxiliar no tratamento de diabéticos é complexo pela duração da doença, comorbidades e limitações pelas manifestações das patologias. Condição sociodemográfica influencia a compreensão do estado de saúde e doença, na adoção das mudanças de estilo de vida e terapêuticas.

Palavras-chave: Assistência Ambulatorial. Diabetes Mellitus. Doença Crônica. Enfermagem.

\section{ABSTRACT}

Noncommunicable Diseases, especially Diabetes Mellitus (DM), are a serious public health problem, being the nursing consultation (NC) an instrument used to control the disease and its diseases. A study based on NC experiences in patients with DM in outpatient clinic of tertiary level health establishment, university hospital capital of southern Brazil. Knowledge of the social, economic and health care of the patients being treated is a way of assisting nurses in obtaining better response to combinations and guidelines improving disease control and increasing the quality of life of the patient. Had as objective, to characterize the sociodemographic and health profile of diabetic patients seen in a nursing consultation (NC) at a teaching hospital outpatient clinic. Character quantitative study, cross-sectional design. The study sample consisted of adult diabetic patients with first NC appointment in the Diabetic Adult Nursing outpatient clinic between 2016 and 2017. Data analysis showed predominance of females, white, married, born outside of the state capital metropolitan area, resident in the capital and region, low education and witch many missing appointments. In health, it was observed: comorbidities, glycemic and glycated hemoglobin levels outside the therapeutic goals. It is concluded the challenge in assisting the treatment of diabetics is complex due to the duration of the disease, comorbidities and limitations due to the manifestations of the pathologies. Sociodemographic condition influences the understanding of health and disease status in the adoption of lifestyle and therapeutic.

Keywords: Chronic Disease. Diabetes Mellitus. Nursing. Outpatient Care. 


\section{INTRODUÇÃO}

O diabetes mellitus (DM) ocupa o sétimo lugar no ranking das 10 doenças que mais causaram óbitos em 2016, com 1.600.000 mortes (OMS, 2018). No Brasil, o DM e a Hipertensão Arterial Sistêmica são a primeira causa de mortalidade e de hospitalizações, sendo apontadas como responsáveis por mais da metade dos diagnósticos primários em pessoas com insuficiência renal crônica submetidas à diálise no Sistema Único de Saúde (OPAS, 2010).

O Diabetes se caracteriza como um grupo de doenças metabólicas caracterizadas por níveis elevados de glicose plasmática causada por defeitos na secreção da insulina, da ação da insulina ou de ambos. É uma doença crônica não transmissível, causadora de danos em longo prazo e intimamente ligada à perda da visão (retinopatias), doenças vasculares, insuficiência renal e amputações de membros (ASSOCIAÇÃO AMERICANA DE DIABETES, 2013). Hipertensão e dislipidemia também são achados comuns em pacientes diabéticos (ASSOCIAÇÃO AMERICANA DE DIABETES, 2013).

A grande maioria dos casos de diabetes se enquadra em dois grupos: o diabetes tipo 1(DM1), determinado por uma deficiência absoluta de secreção de insulina, em geral causada por uma reação autoimune que leva a destruição das ilhotas pancreáticas; o diabetes tipo 2 (DM2), causado por uma combinação de resistência à ação da insulina e uma resposta de secreção inadequada de insulina. Níveis glicêmicos altos o suficiente para causar danos graves a diversos órgãos e sistemas podem ocorrer mesmo na ausência de sintomas clínicos aparentes, não raro quando do surgimento de sintomas da doença como poliúria, polidipsia, perda de peso e aumento do apetite a mesma já cursando a um longo tempo e determinando danos graves (AMERICAN DIABETES ASSOCIATION, 2014). Cerca de $90 \%$ dos casos de diabetes diagnosticados são de Diabetes Mellitus tipo 2 (DM2) (FONTBONNE, 2013).

A mudança de perfil epidemiológico e demográfico causa uma profunda crise nos sistemas de atenção à saúde vigentes (MENDES, 2010). Em países subdesenvolvidos, essa crise torna-se particularmente mais grave, devido à tripla carga de doenças infecciosas, ligadas à reprodução e desnutrição ainda não superadas, somados ao surgimento do novo desafio das doenças crônicas não transmissíveis (DCNT) e o aumento da mortalidade por causas externas (FRENK, 2006).

Neste contexto de mudança de perfil epidemiológico e demográfico a consulta de enfermagem (CE) torna-se um instrumento eficaz e importante dentro da necessidade de adaptação do sistema de saúde vigente ao novo perfil epidemiológico e demográfico somado a sua possibilidade de inserção nos vários níveis do sistema de saúde (CESSE, 2007; MENDES, 2010; BRASIL, 2019).

O Conselho Federal de Enfermagem (COFEN), por meio da Lei ${ }^{\circ} 7.498 / 86$, de 25 de junho de 1986 define a CE como atividade privativa do enfermeiro. Já a Resolução do COFEN-358/2009 caracteriza a mesma como sendo o processo de enfermagem realizado em instituições prestadoras de serviços ambulatoriais de saúde, domicílios, escolas, associações comunitárias, entre outros. A CE organiza-se em cinco etapas inter-relacionadas, interdependentes e recorrentes: coleta de dados de Enfermagem (ou Histórico de Enfermagem), diagnóstico de enfermagem, planejamento de Enfermagem, implementação e avaliação (COFEN, 1986; COFEN, 2009). Há que se destacar que por suas características próprias a $\mathrm{CE}$ tem grande factibilidade nas inúmeras situações e realidades por estar centrada principalmente em tecnologias leves e leve-duras (MERHY, 2002; COFEN, 2009).

Tais tecnologias classificam-se em: leves (ligadas às relações humanas/interpessoais), leveduras (saberes estruturados) e duras (equipamentos) (MERHY, 2002). As tecnologias leves na CE têm influência nos processos de organização do trabalho e nas relações interpessoais, tornando a abordagem dos pacientes mais eficaz e resolutiva. Existe co-responsabilização pelo cuidado através dos acordos e combinações entre o enfermeiro e o paciente, o cuidado se torna individualizado e cria-se o vínculo profissional-usuário o que auxilia na continuidade do cuidado (CARLOS, 2017). 
Nesta perspectiva, o conhecimento da situação social, econômica e de saúde dos pacientes atendidos é mais uma forma de auxiliar o enfermeiro na obtenção de melhores respostas às combinações e orientações, auxiliando na melhora do controle da doença e assim na sua qualidade de vida. Com isso, foi proposto este estudo visando caracterizar o perfil sociodemográfico e de saúde de pacientes diabéticos atendidos em consulta de enfermagem em ambulatório de um hospital universitário.

\section{METODOLOGIA}

Trata-se de estudo de abordagem quantitativa de delineamento transversal. A amostra selecionada e a determinação dos parâmetros do fator em estudo e do desfecho foram analisadas em um ponto do tempo, sem acompanhamento posterior (NEWMAN, 2008; POLIT, 2011).

O estudo foi desenvolvido no Ambulatório do Hospital de Clínicas de Porto Alegre (HCPA), na agenda de CE à pacientes portadores de DM, denominada de EAD - grade 23442 (Enfermagem Adulto Diabético). O HCPA é uma empresa pública de direito privado, integrante da rede de hospitais do Ministério da Educação, vinculado academicamente à Universidade Federal do Rio Grande do Sul - UFRGS (HOSPITAL DE CLÍNICAS DE PORTO ALEGRE, 2017). A referida agenda de atendimento destina-se ao ensino de alunos de graduação da Escola de Enfermagem da UFRGS.

A amostra do estudo foi constituída por pacientes adultos diabéticos com as faixas etárias de 40-59 anos, 60-79 anos e 80 anos ou mais, que tiveram a primeira consulta marcada na agenda de CE a pacientes portadores de DM, denominada de EAD - grade 23442 no período de 2016 a 2017. A agenda é focada para pacientes com diagnóstico de DM2. Dos quarenta e dois pacientes que tiveram a primeira consulta marcada no período estudado dois foram excluídos do estudo por tratar-se de adolescentes.

Os dados foram coletados retrospectivamente compreendendo os anos de 2016 e 2017 a partir de dados secundários de sistema IG do HCPA, por meio de solicitação de query, bem como nos prontuários eletrônicos. Foi utilizado um instrumento projetado especificamente para este fim contendo as variáveis de interesse: dados sociodemográficos, data da primeira consulta, número de consultas realizadas, número de faltas em consultas, presença de acompanhante, índice de massa corporal, glicemia capilar, perfil lipídico e glicêmico, pressão arterial, número de medicamentos em uso, número de refeições diárias, prática de atividade física, cuidados com os pés, uso de insulina e realização do rodízio para aplicação de insulina.

Os dados coletados foram organizados em planilhas Excel utilizando-se o programa Action Stat 3 para os cálculos estatísticos. Utilizou-se a análise descritiva para descrever as variáveis de interesse. A média e desvio padrão foram utilizados para a descrição das variáveis quantitativas com distribuição normal e as variáveis quantitativas com distribuição assimétrica serão descritas pela mediana e intervalo interquartil. As variáveis categóricas foram descritas através das frequências absolutas e relativas. O teste que foi utilizado para verificar a normalidade das variáveis quantitativas foi o Shapiro-Wilk. Os dados estão apresentados por meio de tabelas.

Os pesquisadores do projeto se comprometem a manter o sigilo dos dados coletados nos prontuários. As informações foram utilizadas única e exclusivamente com finalidade científica, preservando-se integralmente o anonimato dos participantes. O projeto foi submetido à Comissão de Pesquisa da Escola de Enfermagem (COMPESQ) da Universidade Federal do Rio Grande do Sul (UFRGS) $n^{\circ}$ do protocolo de aprovação 170686, aceito em 28/12/2017. Foram respeitados os aspectos éticos para pesquisas em seres humanos de acordo com a Resolução 466/2012 do Conselho Nacional de Saúde (BRASIL, 2012). O risco envolvido no projeto é a quebra de confidencialidade. Para minimizar este risco, os pacientes foram identificados por números, assegurando a preservação de seus nomes. 


\section{RESULTADOS E DISCUSSÃO}

$\mathrm{Na}$ Tabela 1 constam os resultados referentes às distribuições das variáveis sociodemográficas analisadas da amostra total e por sexo.

Tabela 1 - Distribuição das variáveis: gênero, idade, naturalidade, procedência, raça, estado civil, escolaridade, religião, faltas e presenças em consultas

\begin{tabular}{|c|c|c|c|}
\hline & $\begin{array}{c}\text { Feminino } \\
\mathbf{5 5 \%} \\
(\mathbf{n}=\mathbf{2 2})\end{array}$ & $\begin{array}{c}\text { Masculino } \\
45 \% \\
(n=18)\end{array}$ & $\begin{array}{c}\text { Geral } \\
(n=40)\end{array}$ \\
\hline Média de idade (anos) & $55,5 \pm 11,8$ & $57,5 \pm 11,3$ & $56,4 \pm 11,5$ \\
\hline \multicolumn{4}{|l|}{ Naturalidade } \\
\hline Interior & $68,2 \%(15)$ & $55,5 \%(10)$ & $62,5 \%(25)$ \\
\hline *Porto Alegre e região metropolitana & $27,3 \%(6)$ & $38,9 \%(7)$ & $32,5 \%(13)$ \\
\hline Outro estado/país & $4,5 \%(1)$ & $5,5 \%(1)$ & $5 \%(2)$ \\
\hline \multicolumn{4}{|l|}{ Procedência } \\
\hline Interior & $13,6 \%(3)$ & $11,1 \%(2)$ & $12,5 \%(5)$ \\
\hline *Porto Alegre e região metropolitana & $86,4 \%(19)$ & $88,9 \%(16)$ & $87,5 \%(35)$ \\
\hline \multicolumn{4}{|l|}{ **Raça } \\
\hline Branca & $81,8 \%(18)$ & $83,3 \%(15)$ & $82,5 \%(33)$ \\
\hline Negra & $18,9 \%(4)$ & $5,5 \%(1)$ & $12,5 \%(5)$ \\
\hline Parda & $0 \%(0)$ & $11,1 \%(2)$ & $5 \%(2)$ \\
\hline \multicolumn{4}{|l|}{ Estado civil } \\
\hline Solteiro & $40,9 \%(9)$ & $16,7 \%(3)$ & $30 \%(12)$ \\
\hline Casado & $36,4 \%(8)$ & $77,7 \%(14)$ & $55 \%(22)$ \\
\hline Viúvo & $9,1 \%(2)$ & $5,5 \%(1)$ & $7,5 \%(3)$ \\
\hline Separado & $13,6 \%(3)$ & $0 \%(0)$ & $7,5 \%(3)$ \\
\hline \multicolumn{4}{|l|}{ Escolaridade } \\
\hline Analfabeto & $0 \%(0)$ & $5,5 \%(1)$ & $2,5 \%(1)$ \\
\hline Fundamental completo & $22,7 \%(5)$ & $22,2 \%(4)$ & $22,5 \%(9)$ \\
\hline Fundamental incompleto & $40,9 \%(9)$ & $33,3 \%(6)$ & $37,5 \%(15)$ \\
\hline Médio completo & $9,1 \%(2)$ & $22,2 \%(4)$ & $15 \%(6)$ \\
\hline Médio incompleto & $18,2 \%(4)$ & $11,1 \%(2)$ & $15 \%(6)$ \\
\hline Superior completo & $0 \%(0)$ & $0 \%(0)$ & $0 \%(0)$ \\
\hline Superior incompleto & $4,50 \%$ & $5,5 \%(1)$ & $5 \%(2)$ \\
\hline Outro & $4,50 \%$ & $0 \%(0)$ & $2,5 \%(1)$ \\
\hline \multicolumn{4}{|l|}{ Religião } \\
\hline Católica & $50 \%(11)$ & $61,1 \%(11)$ & $55 \%(22)$ \\
\hline Evangélica & $13,6 \%(3)$ & $5,5 \%(1)$ & $10 \%(4)$ \\
\hline Testemunha de Jeová & $0 \%(0)$ & $5,5 \%(1)$ & $2,5 \%$ \\
\hline Não tem religião & $9,1 \%(2)$ & $0 \%(0)$ & $5 \%(2)$ \\
\hline Sem registro & $27,3 \%(6)$ & $27,8 \%(5)$ & $27,5 \%(11)$ \\
\hline Não compareceram a nenhuma consulta & $31,8 \%(7)$ & $38,9 \%(7)$ & $35 \%(4)$ \\
\hline Compareceram a uma consulta ou mais & $68,2 \%(15)$ & $61,1 \%(11)$ & $65 \%(6)$ \\
\hline Faltaram a uma consulta ou mais & $63,3 \%(14)$ & $77,8 \%(14)$ & $70 \%(8)$ \\
\hline Compareceram a todas as consultas marcadas & $36,4 \%(8)$ & $22,2 \%(4)$ & $30 \%(2)$ \\
\hline
\end{tabular}

Nota: ** Não houve registro de indivíduos identificados como pertencentes a outras raças além das listadas na tabela 1 .

Fonte: os autores.

Quanto a ocupação, 14 pacientes (35\%) eram aposentados ou pensionistas, $2(5 \%)$ eram bolsistas estagiários, $2(5 \%)$ trabalhavam com fabricação de roupas, 9 (22,5\%) estavam distribuídos igualmente em várias ocupações e 13 (32,5\%) tinham registrado no prontuário a ocupação como outros. A Tabela 2 traz a distribuição das variáveis do perfil de saúde do estudo. 
Tabela 2 - Distribuição das variáveis: glicemia capilar, pressão arterial sistólica, pressão diastólica, índice de massa corporal, acompanhante na consulta, status nutricional pelo IMC, medicações em uso, uso de insulina, número de refeições ao dia e cuidados com os pés

\begin{tabular}{|c|c|c|c|}
\hline & $\begin{array}{c}\text { Feminino } \\
57,7 \% \\
(n=15)\end{array}$ & $\begin{array}{c}\text { Masculino } \\
42,3 \% \\
(n=11) \\
\end{array}$ & $\begin{array}{l}\text { Geral } \\
(n=26)\end{array}$ \\
\hline Glicemia capilar média na consulta (mg/dl) & $203(135-365)$ & $244,4 \pm 119,4$ & $241,6 \pm 119$ \\
\hline *Pressão arterial sistólica média $(\mathrm{mmHg})$ & $140(120-150)$ & $132,1 \pm 17,2$ & $135 \pm 15,8$ \\
\hline *Pressão arterial diastólica média $(\mathrm{mmHg})$ & $80(80-97,5)$ & $80(75-85)$ & $80(80-90)$ \\
\hline Índice médio de massa corporal $\left(\mathrm{Kg} / \mathrm{m}^{2}\right)$ & $35,1 \pm 9,9$ & $30,6 \pm 4,7$ & $33,2 \pm 8,3$ \\
\hline Média de HDL sérico (mg/dl) & $39 \pm 9$ & $44 \pm 12,3$ & $43(33,5-47,5)$ \\
\hline **Média de LDL sérico $(\mathrm{mg} / \mathrm{dl})$ & $80,2 \pm 25,7$ & $93,5 \pm 30,2$ & $88 \pm 27$ \\
\hline Média de colesterol sérico (mg/dl) & $175,4 \pm 40,4$ & $175,7 \pm 31,6$ & $175,5 \pm 34,8$ \\
\hline Média de triglicerídeos sérico (mg/dl) & 201(159-270,7) & $199,5 \pm 122,4$ & $237,4 \pm 186,1$ \\
\hline Média de glicose sérica em jejum (mg/dl) & $150(127,2-236,5)$ & $182,9 \pm 94,1$ & $211,7 \pm 92$ \\
\hline Média de hemoglobina glicada (\%) & $9,3 \pm 1,9$ & $10,3 \pm 1,7$ & $9,8 \pm 1,8$ \\
\hline \multicolumn{4}{|l|}{ Acompanhante na consulta } \\
\hline Acompanhado & $26,7 \%(4)$ & $27,3 \%(3)$ & $27,3 \%(3)$ \\
\hline Desacompanhado & $73,3 \%(11)$ & $54,5 \%(6)$ & $54,5 \%(6)$ \\
\hline Sem registro & $0 \%(0)$ & $18,2 \%(2)$ & $18,2 \%(2$ \\
\hline \multicolumn{4}{|l|}{ Status nutricional pelo IMC } \\
\hline Eutrofia & $20 \%(3)$ & $9,1 \%(1)$ & $15,4 \%(4)$ \\
\hline Sobrepeso & $13,3 \%(2)$ & $36,4 \%(4)$ & $23,1 \%(6)$ \\
\hline Obesidade 1 & $13,3 \%(2)$ & $36,4 \%(4)$ & $23,1 \%(6)$ \\
\hline Obesidade II & $26,7 \%(4)$ & $18,2 \%(2)$ & $23,1 \%(6)$ \\
\hline Obesidade III & $26,7 \%(4)$ & $0 \%(0)$ & $15,4 \%(4)$ \\
\hline \multicolumn{4}{|l|}{ Número de medicações em uso } \\
\hline Até 4 & $13,3 \%(2)$ & $0 \%(0)$ & $7,7 \%(2)$ \\
\hline 5 ou mais sem registro & $40 \%(6)$ & $63,6 \%(7)$ & $50 \%(3)$ \\
\hline \multicolumn{4}{|l|}{ ***Uso de insulina } \\
\hline Não & $46,7 \%(7)$ & $36,4 \%(4)$ & $42,3 \%(1)$ \\
\hline Sim & $26,7 \%(4)$ & $9,1 \%(1)$ & $19,3 \%(5)$ \\
\hline (Sem registro de qual) & $13,3 \%(2)$ & $0 \%(0)$ & $7,7 \%(2)$ \\
\hline NPH & $6,7 \%(1)$ & $27,3 \%(3)$ & $15,4 \%(4)$ \\
\hline $\mathrm{NPH}+$ regular & $20 \%(3)$ & $27,3 \%(3)$ & $23,1 \%(6)$ \\
\hline Sem registro & $33,3 \%(5)$ & $36,4 \%(4)$ & $34,6 \%(9)$ \\
\hline \multicolumn{4}{|l|}{ Número de refeições por dia } \\
\hline Até 3 & $33,3 \%(5)$ & $18,2 \%(2)$ & $26,9 \%(7)$ \\
\hline 4 a 5 & $46,7 \%(7)$ & $63,6 \%(7)$ & $53,8 \%(14)$ \\
\hline 6 & $6,7 \%(1)$ & $0 \%(0)$ & $3,8 \%(1)$ \\
\hline Outras & $13,3 \%(2)$ & $0 \%(0)$ & $7,7 \%(7)$ \\
\hline Sem registro & $0 \%(0)$ & $18,2 \%(2)$ & $7,7 \%(7)$ \\
\hline \multicolumn{4}{|l|}{ Cuidados com os pés } \\
\hline Sim & $0 \%(0)$ & $0 \%(0)$ & $0 \%(0)$ \\
\hline Não & $13,3 \%(2)$ & $9,1 \%(1)$ & $11,5 \%(3)$ \\
\hline Sem registro & $86,7 \%(13)$ & $90,9 \%(10)$ & $88,5 \%(23)$ \\
\hline
\end{tabular}

Notas: *Cinco pacientes (três mulheres e dois homens) não tinham registro da pressão arterial na evolução da consulta; ** A fração lipídica LDL, esta foi calculada com uso de calculadora específica (BRASIL 2018); **Nenhum registro trazia uso de outra insulina além de NPH e regular, assim como nenhuma evolução analisada trazia a informação sobre realização ou não de rodízio nos locais de aplicação da insulina.

Fonte: os autores.

O levantamento e análise dos dados deste estudo nos permite traçar em linhas gerais um perfil destes pacientes. Falando do contexto sociodemográfico percebemos o predomínio do sexo 
feminino (há $10 \%$ menos indivíduos do sexo masculino na amostra), de raça branca $(82,5 \%)$, casadas (as mulheres aparecem com uma porcentagem 41,3\% menor em comparação aos homens), com baixa escolaridade (nenhuma com nível superior completo e 37,5\% apenas com ensino fundamental incompleto), de religião católica, natural do interior e residente na região metropolitana da capital; a idade dos pacientes mostra grande variação.

Em relação ao comparecimento às consultas e vinculação ao serviço o grupo analisado tem grande número de faltas às $\mathrm{CE}$. $70 \%$ faltaram a uma ou mais consultas no período, sendo que destes $35 \%$ não realizaram nem mesmo a primeira consulta apesar de agendada. O grupo do sexo feminino mostra frequência 14,2\% maior de comparecimento, número $14,5 \%$ menor de faltas em comparação ao do sexo masculino. A maioria $(65,4 \%)$ comparece desacompanhada à CE.

Quanto ao controle do DM2, o perfil apontou pacientes dislipidêmicos, com o DM2 não controlado, peso elevado, em uso de mais de 5 medicamentos (destaque para a insulina), níveis de pressão arterial elevados, fazendo de 4 a 5 refeições ao dia e sem rotina adequada de exercícios físicos. A hemoglobina glicada, cuja meta é por volta de $7 \%$ em adultos e entre 7,5\% e 8,5\% em idosos, tem média na amostra de 9,8\% com uma média ainda maior no sexo masculino (10,3\%). Quanto à glicemia de jejum que tem como alvo $<100 \mathrm{mg} / \mathrm{dl}$, a média é de $211,7 \mathrm{mg} / \mathrm{dl}$, ou seja, mais que o dobro do desejado (SOCIEDADE BRASILEIRA DE DIABETES, 2017b).

Neste estudo, constatou-se que $50 \%$ do total da amostra faz uso de cinco ou mais medicamentos ao mesmo tempo (o que na verdade totaliza $86,7 \%$ dos pacientes que possuíam o registro do número de medicações em uso na evolução da consulta).

Dos 26 pacientes com registro em prontuário quanto à realização de atividade física 53,8\% declararam não realizá-la, e 34,6\% relataram realizá-la, desses, apenas $11,5 \%$ contavam com o registro de tempo (minutos) por semana em que realizavam o exercício e destes apenas 3,8\% (um paciente) relatou fazer exercício físico por tempo igual ou superior a 150 minutos por semana.

Segundo a classificação da OMS, $61,6 \%$ da amostra analisada encontra-se obesa. A obesidade, excesso de gordura corporal e suas consequências depende não somente da quantidade absoluta de gordura, mas também da sua distribuição. As complicações incluem, entre outras, a síndrome metabólica, osteoratrite e apneia obstrutiva do sono (YOUDIM, 2018).

A média de idade dos pacientes é de 56,4 anos, 3,6 anos antes do que a Organização Mundial da Saúde (OMS) considera a idade para ser considerado idoso em um país em desenvolvimento como o Brasil (OMS, 2002). Isso corrobora outros estudos sobre o DM2, demonstrando manifestação mais precoce da doença na população e com maior gravidade (LASCAR et al., 2018). Há um número cada vez maior de jovens com DM2 e sabe-se que o tempo de duração à doença leva a um aumento considerável no risco de complicações crônicas. Existem indícios, também, de que a DM2 de início precoce, antes dos 40 anos, tem uma apresentação mais agressiva, com surgimento prematuro de complicações graves. Complicações que podem levar a redução ou perda da capacidade produtiva e ao aumento da carga em saúde pública (LASCAR et al., 2018). Somando-se a transição demográfica, pela qual o Brasil passa, e a relação direta do processo de envelhecimento com o surgimento de DCNT (GOTTLIEB et al., 2011), pode-se compreender a heterogeneidade etária da amostra analisada (desvio padrão amostral de mais de 11 anos).

Via de regra após diagnóstico inicial o DM2 é tratado baseado em medidas de mudança de estilo de vida e uso de hipoglicemiantes orais. Novos estudos, porém, mostram que a insulina é aliada importante e deve ser usada mais precocemente e com mais frequência no controle da DM2, visto que o caráter crônico e progressivo da doença leva a ineficácia da terapia baseada em hipoglicemiantes orais no controle dos níveis glicêmicos. Os agravos causados por uma glicemia constantemente fora do alvo terapêutico superam e muito os possíveis efeitos indesejados da terapia com insulina (DORES, 2013; RÉA, 2014).

O grande número de pacientes em uso de insulina na amostra vem ao encontro das recomendações da Diretriz da Sociedade Brasileira de Diabetes (SBD) 2017/2018 sobre a insulinoterapia na DM2, onde a mesma aparece como terapia indicada para entrar no esquema 
terapêutico de forma rotineira ou transitória em variadas situações, até mesmo logo ao diagnóstico nos casos que apresentam alto grau de descompensação metabólica (SOCIEDADE BRASILEIRA DE DIABETES, 2017b). Vale ressaltar que as mudanças no estilo de vida se mantêm como recomendação para todos os casos diagnosticados de DM2 (SOCIEDADE BRASILEIRA DE DIABETES, 2017a).

Entretanto, mesmo com esse número de indivíduos em uso de ao menos um tipo de insulina, as médias de glicemia de jejum e hemoglobina glicada da amostra analisada mostram-se bastante acima das metas terapêuticas da SBD. A polimedicação é o uso simultâneo e de forma crônica de fármacos para doenças e sintomas distintos pelo mesmo indivíduo, prática cada vez mais frequente que leva a um aumento a probabilidade de reações adversas ao medicamento, interações medicamentosas e diminuição da adesão ao tratamento (ROSA, CAMARGO, 2014). Na amostra, o uso de duas a nove aparece de forma acentuada na amostra. Segundo Viera (2015), é mais apropriada a utilização da definição que a considera como a utilização de pelo menos um fármaco não indicado (VIEIRA, 2015). A polimedicação se torna um problema de saúde pública à medida que onera o Estado (que precisa fornecer esses medicamentos pela rede pública de saúde) e que leva os pacientes, idosos em especial, a ter dificuldades para a utilização dos medicamentos, seja pela dificuldade de acesso (deslocar-se para retirar/adquirir os medicamentos) e, principalmente, a dificuldade de compreender a prescrição (ROSA; CAMARGO, 2014).

A questão cultural pode ser um fator predisponente, a dificuldade em realizar as mudanças necessárias na questão alimentar para melhor controle da obesidade e por consequência das outras doenças ligadas ao quadro de peso excessivo. Os pacientes em sua maioria têm origem no interior do Rio Grande do Sul e, no momento, residem na capital e área metropolitana. Valores e hábitos alimentares da dieta dessa vivência no interior, lida aqui como rural e ativa, não conseguem ser modificadas de forma adequada na transição para a vida na cidade, urbana e sedentária, levando ao ganho de peso (ALVES; COLAÇO, 2017). O baixo número de pacientes que realizam atividade física regular, na qual pacientes diabéticos têm como recomendação atividade aeróbica moderada por 150 minutos por semana, também podem ter influência sobre esse quadro de obesidade e sobrepeso na amostra (SOCIEDADE BRASILEIRA DE DIABETES, 2017b).

A síndrome metabólica é um conjunto de distúrbios metabólicos. Essa ocorre quando uma pessoa tem três ou mais fatores, como a obesidade/sobrepeso, DM2, dislipidemia e hipertensão. A síndrome metabólica é uma condição grave de saúde que afeta cerca de $23 \%$ dos adultos e os coloca em maior risco de doenças cardiovasculares do que qualquer fator isolado sozinho (AMERICAN HEART ASSOCIATION, 2018).

\section{CONCLUSÃO}

Ao encerrar este estudo concluímos que o desafio da $\mathrm{CE}$ em auxiliar no tratamento dos pacientes diabéticos é complexo pela própria característica de longa duração das DCNT, por recebermos pacientes já com inúmeras comorbidades e as limitações surgidas pelas manifestações destas patologias. A condição sociodemográfica também é fator a ser considerado com cuidado no planejamento e implementação das abordagens de cuidado a estes pacientes, pois tem influência direta na compreensão do estado de saúde e doença pelo próprio indivíduo, e na adoção das mudanças de estilo de vida e das terapêuticas farmacológicas para um controle e tratamento adequados do diabetes.

Ao olhar para o perfil traçado no presente estudo levantam-se inúmeras questões para o resultado obtido e que talvez possam ser utilizados em novas pesquisas: Há ligação entre as complicações articulares causadas pela obesidade com o reduzido número de pacientes que realizam atividade física? A polimedicação destes pacientes é realmente necessária e benéfica? Quais os reais motivos para não atingir as metas terapêuticas de controle glicêmico? Os pacientes conseguem seguir as prescrições de medicamentos corretamente? 
Sugere-se também a criação de instrumento específico para as CE de modo a não se perderem dados importantes para o acompanhamento do paciente e mantendo sua continuidade (o campo de estudo da amostra recebe novos estudantes semestralmente que realizam as CE sob orientação de preceptora).

Além disso, sugere-se também a realização de novos estudos com o mesmo objetivo, com amostra maior e coleta direta dos dados (com o paciente na consulta e não recuperados dos registros do prontuário) para confirmação dos dados encontrados neste estudo.

\section{REFERÊNCIAS}

ALVES, L. F.; COLAÇO, J. H. L. Comer na cidade: mudanças alimentares, obesidade e êxodo rural na cidade de Goiânia, Goiás. In: COLAÇO, J. H. L.; BARBOSA, F. A. C.; ROLIM, T. P. B. (Org.). Cidades e consumo alimentar. Goiânia: Imprensa Universitária Digital, p. 121-143, 2017. Disponível em: <https://www.cegraf.ufg.br/up/688/o/ebook_cidades_consumo2.pdf >. Acesso em: 24 nov. 2018.

AMERICAN DIABETES ASSOCIATION. Diagnosis and classification of diabetes mellitus. Diabetes Care, v. 37, n. 1 Supl., p. 81-90. 2014. Acesso em: 23 nov. 2017.

AMERICAN HEART ASSOCIATION. About Metabolic Syndrome. Disponível em: <http://www.heart.org/en/health-topics/metabolic-syndrome/about-metabolic-syndrome>. Acesso em: 25 nov. 2018.

ASSOCIAÇÃO AMERICANA DE DIABETES. Diagnosis and Classification of Diabetes Mellitus. Diabetes Care, v. 37, n. 1, p. 81-90. 2013. Disponível em:<http://care.diabetesjournals.org/content/37/Supplement_1/S81>. Acesso em: 30 out. 2017.

BRASIL. Conselho Nacional de Saúde. 2012. Disponível em:<http://conselho.saude.gov.br/web_comissoes/conep/aquivos/resolucoes/resolucoes >.htm. Acesso em: 27 jun. 2019.

BRASIL. Ministério da Saúde. Secretaria de Vigilância em Saúde. Coordenação-Geral de Desenvolvimento da Epidemiologia em Serviços. 3.ed. Volume único. Guia de Vigilância em Saúde, Coordenação-Geral de Desenvolvimento da Epidemiologia em Serviços. Brasília: Ministério da $\quad$ Saúde, 2019. $\quad$ Disponível em: http://bvsms.saude.gov.br/bvs/publicacoes/guia_vigilancia_saude_3ed.pdf Acesso: 06 jul 2019

CARLOS, D. M. As Tecnologias leves no contexto da Estratégia De Saúde da Família: (Re) Organização do trabalho. Conduta terapêutica no diabetes tipo 2: Algoritmo SBD 2017, 2017. Disponível em: https://www.diabetes.org.br/profissionais/images/2017/POSICIONAMENTOOFICIAL-SBD-02-2017-ALGORITMO-SBD-2017.pdf. Acesso em: 25 nov.2018.

CESSE, E. A. P. Epidemiologia e Determinantes Sociais das Doenças Crônicas Não Transmissíveis no Brasil. Fundação Oswaldo Cruz Centro de Pesquisas Aggeu Magalhães, Recife, 2007. Disponível em: https://www.arca.fiocruz.br/bitstream/icict/3905/2/000006.pdf. Acesso em: 25 nov 2018

CONSELHO FEDERAL DE ENFERMAGEM. Lei N 7.498/86 de 25 de junho de 1986. 1986. Disponível em: <http://www.cofen.gov.br/lei-n-749886-de-25-de- junho-de-1986_4161.html>. Acesso em: 27 jun. 2019. 
CONSELHO FEDERAL DE ENFERMAGEM. Resolução 358/2009. 2009. Disponível em: <http://www.cofen.gov.br/resoluo-cofen-3582009_4384.html>.Acesso em: 27 jun. 2019.

DORES, J. Insulinoterapia na diabetes mellitus tipo 2. Revista Portuguesa de Cardiologia, Portugal, v. 32, n. 1, p. 25-31, 2013. Disponível em: <http://www.revportcardiol.org/pt-pdfS0870255113700441>. Acesso em: 24 nov. 2018.

FONTBONNE, A. Diabetes tipo 2: um cenário preocupante. Brasil, 2013. Disponível em: <http://dssbr.org/site/opinioes/diabetes-tipo-2-um-cenario-preocupante/>. Acesso em: 23 jun. 2016.

FRENK J. Bridging the Divide: Comprehensivere Form to Improve Health in Mexico. Nairobi: Commissionon Social Determinants of Health, 2006. Disponível em: <http://www.who.int/social _determinants/resources/frenk.pdf>. Acesso em: 23 out. 2017.

GOTTLIEB, M. G. V. et al. Envelhecimento e longevidade no Rio Grande do Sul: um perfil histórico, étnico e de morbi-mortalidade dos idosos. Revista Brasileira de Geriatria e Gerontologia, v. 14, n. 2, p. 365-380, 2011. Disponível em: <http://www.scielo.br/scielo.php?script=sci_arttext\&pid=S1809-98232011000200016\&lng=pt\& nrm=iso>. Acesso em: 24 nov. 2018.

HOSPITAL DE CLÍNICAS DE PORTO ALEGRE. Relatório de gestão do exercício de 2016. Porto Alegre, 2017. Disponível em: <https://www2.hcpa.edu.br/downloads/Comunicacao/relatorio_ gesto_2016_-_tcu_-_final.pdf>. Acesso em: 30 out. 2017.

LASCAR, N. et al. Type 2 diabetes in adolescents and young adults. The Lancet Diabetes \& Endocrinology, v. 6, n. 1, p. 69-80, 2018. Disponível em: <https://www.sciencedirect.com/science/ article/pii/S2213858717301869>. Acesso em: 24 nov. 2018.

MENDES, E. V. As redes de atenção à saúde. Ciência \& Saúde Coletiva, v. 15, n. 5, p. 22972305, 2010. Disponível em: <http://www.scielo.br/scielo.php?script=sci_arttext\&pid=S1413$81232010000500005 \& \operatorname{lng}=$ en\&nrm=iso>. Acesso em: 23 out. 2017.

MERHY, E. E. Saúde: a cartografia do trabalho vivo. 3.ed. São Paulo: Editora Hucitec, 2002. Disponível: <http://www.scielo.br/pdf/csp/v24n8/23.pdf>. Acesso em: 30 out. 2017.

NEWMAN, T. B. et al. Delineando estudos transversais e de caso-controle. In: HULLEY, S. B. et al. Delineando a pesquisa clínica: uma abordagem epidemiológica. 3.ed. Porto Alegre: Artmed, 2008. p. 127-144.

ORGANIZAÇÃO MUNDIAL DA SAÚDE. Active ageing: a policy framework. Genebra, 2002. Disponível em: <http://apps.who.int/iris/bitstream/handle/10665/67215/WHO_NMH_NPH_02.8. pdf?s equence $=1 \&$ isAllowed $=\mathrm{y}>$. Acesso em: 23 nov. 2018.

ORGANIZAÇÃO MUNDIAL DA SAÚDE. 10 principais causas de morte. Genebra, 2018. Disponível em: <http://www.who.int/en/news-room/fact- sheets/detail/the-top-10-causes-of-death> Acesso em: 28 out. 2018.

ORGANIZAÇÃO PAN-AMERICANA DA SAÚDE. Linhas de cuidado: hipertensão arterial e diabetes. 1.ed. Brasília, 2010. Disponível em: <http://bvsms.saude.gov.br/bvs/publicacoes/linhas_ cuidado_hipertensao_diabetes.pdf>. Acesso em: 24 jun. 2017. 
POLIT, D. F, BECK, C. T. Fundamentos de pesquisa em enfermagem: avaliação de evidência para a prática da enfermagem. 7. ed. Porto Alegre: Artmed, 2011.

RÉA, R. Insulinização no diabetes tipo 2: quando e como? In. E-book Diabetes na prática clínica. Capítulo 4, 2014. Disponível em: <https://www.diabetes.org.br/ebook/component/k2/item/56insulinizacao-no-diabetes- tipo-2> Acesso em:25 nov.2018

ROSA, G. R.; CAMARGO, E. A. F. Polimedicação em idosos. Interciência \& Sociedade - v. 3, n. 2, p. 72-78, 2014. Disponível em: <https://intercienciaesociedade.francomontoro.com.br/colecao/ impressa/v3_n2/polimedicacao_idosos.pdf>Acesso em: 25 nov. 2018.

SOCIEDADE BRASILEIRA DE DIABETES. Conduta terapêutica no diabetes tipo 2: algoritmo SBD 2017. Brasil, 2017. Disponível em: <https://www.diabetes.org.br/profissionais/ images/2017/POSICIONAMENTO-OFICIAL-SBD-02-2017-ALGORITMO-SBD-2017.pdf>. Acesso em: 25 nov. 2018

SOCIEDADE BRASILEIRA DE DIABETES. Diretrizes da Sociedade Brasileira de Diabetes 2017-2018. Brasil, 2017b. Disponível em: <https://www.diabetes.org.br/profissionais/images/2017/ diretrizes/diretrizes-sbd-2017-2018>. Acesso em: 25 nov. 2018.

VIEIRA, P. A. F. Polimedicação no idoso. 2015. 94f. Dissertação (Mestrado) - Curso de Medicina, Universidade de Coimbra, Coimbra, 2015. Disponível em: <https://core.ac.uk/download/pdf/ 43586756.pdf>. Acesso em: 23 nov. 2018.

YOUDIM, A. Obesidade. Manual MSD, 2018. Disponível em: <https://www.msdmanuals.com/ptbr/profissional/dist\%C3\%BArbios-nutricionais/obesidade-e-s\%C3\%ADndromemetab\%C3\%B3lica/obesidade>. Acesso em: 24 nov. 2018. 